\title{
Migratory gene expression signature predicts poor patient outcome: Are cancer stem cells to blame?
}

\author{
Max S Wicha* \\ See related research by Patsialou et al., http://breast-cancer-research.com/content/14/5/R139
}

\begin{abstract}
Breast cancer metastasis accounts for the majority of deaths from this disease. In the previous issue of Breast Cancer Research, Patsialou and colleagues used a novel in vivo invasion assay to capture migrating breast cancer cells and demonstrate that the gene expression signature of these cells predicts breast cancer metastasis in a large cohort of patients. Furthermore, specific genes identified play a functional role in the invasion of MDA-MB-231 breast cancer cells and in patient-derived breast tumors. These genes regulate pathways known to be induced in invasion and metastases and play an important role in the regulation of cancer stem cells.
\end{abstract}

The majority of cancer deaths result from systemic metastases, the development of which requires the coordinate regulation of complex genetic programs. Patsialou and colleagues [1] recently reported that the expression of genes related to these programs predicted the development of metastases in patients with breast cancer. Tumor metastasis is dependent upon, and orchestrated by, the genetic and epigenetic state of cancer cells and the interaction of these cells with the tumor and host microenvironments [2]. To elucidate the regulatory pathways involved in tumor invasion and metastasis, Patsialou and colleagues [3] previously developed an in vivo model in which microneedles containing a chemoattractant such as epidermal growth factor were placed in tumors in live tumor-bearing mice. In the previous issue of Breast Cancer Research, the same group used this system to isolate migratory cell populations in highly metastatic MDA-MB-231 breast cancer xenografts [1]. They used gene expression analysis of these migrated

*Correspondence: mwicha@umich.edu

University of Michigan Comprehensive Cancer Center, 6302 CC, 1500 E. Medical Center Dr., Ann Arbor, MI 48109-5942, USA cells to define a 'human invasion signature' (HIS). Interestingly, they demonstrated that HIS was predictive of risk of breast cancer metastasis in large cohorts of patients. This association was seen across all molecular subtypes of breast cancer and was independent of known prognostic factors. In addition to the clinical correlates of HIS, this signature identifies genes known to be involved in key processes of metastasis, including embryonic tissue development, cellular movement, and DNA replication and repair.

There is now substantial evidence that breast cancers are hierarchically organized and driven by a fraction of cells that display stem cell properties. These breast cancer stem cells (BCSCs), by virtue of their relative resistance to chemotherapy and radiation therapy, may contribute to treatment resistance [4]. Furthermore, a number of groups, including our own [5], have demonstrated that BCSCs mediate invasion and metastasis both in vitro and in mouse models. Other studies have demonstrated a correlation between expression of BCSC markers such as $\mathrm{CD} 44^{+} / \mathrm{CD} 24^{-}$or aldehyde dehydrogenase or gene expression profiles of enriched BCSCs with the development of metastasis and poor clinical outcome in patients with breast cancer [6]. Although these studies suggest an important relationship between CSCs and the metastatic phenotype, the gene programs in BCSCs responsible for mediating these behaviors are poorly defined.

To identify genes important in the invasive process, Patsialou and colleagues examined the functional role of a number of genes identified in the HIS. They used both small interfering RNAs (siRNAs) and inhibitory antibodies to demonstrate a role for specific HIS genes in the tumor invasive phenotype. Using these techniques, they demonstrated a role for transforming growth factor-beta (TGF $\beta$ ), interleukin-8 (IL-8), PTPN11, and NP1 in mediating invasion of MDA-MB-231 cells. Interestingly, all of these genes have been reported to be important regulators of CSCs. TGF $\beta$ has been shown to regulate the self-renewal of CSCs in several tumor types, and, most recently, this cytokine was shown to specifically regulate BCSCs in claudin ${ }^{\text {lo }}$ breast cancers [7], the subtype that is represented by MDA-MB-231 cells. Our group reported 
that the cytokine IL-8 mediates BCSC self-renewal and that the IL-8 receptor CXCR1 is preferentially expressed in BCSCs [5]. Inhibition of this receptor reduces the BCSC population in mouse xenografts, inhibiting tumor growth and metastasis [8]. PTPN11 encodes the tyrosine phosphatase SHP2, which was recently shown to promote breast cancer progression through expansion of the BCSC population [9]. This occurs via activation of the zinc finger E-box-binding homeodomain ZEB1 protein, which in turn represses Let 7 microRNA, an important BCSC regulator [10]. Finally, NPM1 (nucleophosmin) was reported to upregulate the gene profile involved in hematopoietic stem cell regulation [11].

What is the relationship of genes included in HIS and the CSC phenotype? Interestingly, Patsialou and colleagues [1] reported a significant correlation of HIS with gene signatures associated with epithelial-mesenchymal transition (EMT). EMT has been implicated in tissue invasion and metastasis, and, more recently, similarities between EMT and CSCs were described [12]. However, Patsialou and colleagues found a negative correlation between HIS and previously published tumor-initiating cell (TIC) signatures. Genes that were upregulated in TIC were downregulated in HIS, while genes downregulated in TIC were enriched in HIS [1]. This apparent discrepancy may be explained by the existence of alternative CSC states [13]. We have previously proposed that CSCs may exist in either an EMT mesenchymal-like state characterized by tissue invasion and motility or an MET epithelial-like state associated with self-renewal. EMT-like CSCs are preferentially located at the tumor invasive front, where they invade blood vessels and travel to distant metastatic sites forming micrometastases. This model is consistent with reports that circulating tumor cells and micrometastases are enriched for the EMT BCSC markers CD44 /CD24- but that these cells are largely quiescent as evidenced by a lack of $\mathrm{Ki} 67$ expression [14]. The tumor microenvironment at metastatic sites may induce a transition of these EMT CSCs to a more epithelial mesenchymal-epithelial transition (MET) state characterized by aldehyde dehydrogenase expression, in which they self-renew and differentiate, generating the bulk tumor populations that constitute clinically relevant metastases. This model predicts that gene expression profiles of EMT and MET CSCs may be highly divergent and in fact negatively correlated with regard to genes involved in EMT/MET state transitions. This model provides a potential explanation for the negative correlations of HIS and TIC gene signatures reported by Patsialou and colleagues [1]

The studies by Patsialou and colleagues represent an important advance in understanding elements of the metastatic cascade. However, these studies have several limitations. Since they rely primarily on a single claudin low cell line model, it is unclear how widely the HIS applies to other molecular subtypes of breast cancer. Although the HIS was an independent prognostic predictor across the different molecular subtypes of breast cancer, it remains unclear whether similar metastatic programs are involved in mediating metastasis in these other subtypes. Different breast cancer subtypes have different tropisms for sites of metastasis (for example, estrogen receptor-positive tumors frequently metastasize to bone, HER2-positive breast cancer to brain and TN breast cancer to liver and lung). Furthermore, recurrence occurs with markedly different latencies for these subtypes, so, clearly, additional studies are needed to understand the mechanisms which account for these critical differences. Furthermore, additional studies will be required to delineate the relationship between HIS and CSC regulatory genes. These studies have important implications for understanding the molecular basis of tumor invasion and metastasis and have significant clinical implications. Targets identified in these and similar studies may provide new opportunities to develop therapies for metastatic breast cancer.

\section{Abbreviations}

BCSC, breast cancer stem cell; CSC, cancer stem cell; EMT, epithelialmesenchymal transition; HIS, human invasion signature; IL-8, interleukin-8; MET, mesenchymal-epithelial transition; TGF $\beta$, transforming growth factorbeta; TIC, tumor-initiating cell.

\section{Competing interests}

The author is a co-founder of and holds equity in OncoMed Pharmaceuticals (Redwood City, CA, USA), is a scientific advisor for Verastem (Cambridge, MA, USA) and Paganini Biopharma (Encino, CA, USA), and receives research support from Dompé farmaceutici (Milan, Italy).

Published: 15 November 2012

\section{References}

1. Patsialou A, Wang Y, Lin J, Whitney K, Goswami S, Kenny PA, Condeelis JS: Selective gene-expression profiling of migratory tumor cells in vivo predicts clinical outcome in breast cancer patients. Breast Cancer Res 2012, 14:R139.

2. Polyak K, Haviv I, Campbell IG: Co-evolution of tumor cells and their microenvironment. Trends Genet 2009, 25:30-38.

3. Patsialou A, Wyckoff J, Wang Y, Goswami S, Stanley ER, Condeelis JS: Invasion of human breast cancer cells in vivo requires both paracrine and autocrine loops involving the colony-stimulating factor-1 receptor. Cancer Res 2009, 69:9498-9506.

4. Morrison R, Schleicher SM, Sun Y, Niermann KJ, Kim S, Spratt DE, Chung CH, Lu B: Targeting the mechanisms of resistance to chemotherapy and radiotherapy with the cancer stem cell hypothesis. J Onco/ 2011, 2011:941876.

5. Charafe-Jauffret E, Ginestier C, lovino F, Wicinski J, Cervera N, Finetti P, Hur MH, Diebel ME, Monville F, Dutcher J, Brown M, Viens P, Xerri L, Bertucci F, Stassi G, Dontu G, Birnbaum D, Wicha MS: Breast cancer cell lines contain functional cancer stem cells with metastatic capacity and a distinct molecular signature. Cancer Res 2009, 69:1302-1313.

6. Ginestier C, Hur MH, Charafe-Jauffret E, Monville F, Dutcher J, Brown M, Jacquemier J, Viens P, Kleer CG, Liu S, Schott A, Hayes D, Birnbaum D, Wicha MS, Dontu G: ALDH1 is a marker of normal and malignant breast stem cells and a predictor of poor clinical outcome. Cell Stem Cell 2007, 1:555-567.

7. Bruna A, Greenwood W, Le Quesne J, Teschendorff A, Miranda-Saavedra D, Rueda OM, Sandoval JL, Vidakovic AT, Saadi A, Pharoah P, Stingl J, Caldas C: TGFbeta induces the formation of tumour-initiating cells in claudin(low) 
breast cancer. Nat Commun 2012, 3:1055.

8. Ginestier C, Liu S, Diebel ME, Korkaya H, Luo M, Brown M, Wicinski J, Cabaud O, Charafe-Jauffret E, Birnbaum D, Guan JL, Dontu G, Wicha MS: CXCR1 blockade selectively targets human breast cancer stem cells in vitro and in xenografts. J Clin Invest 2010, 120:485-497.

9. Aceto N, Sausgruber N, Brinkhaus H, Gaidatzis D, Martiny-Baron G, Mazzarol G, Confalonieri S, Quarto M, Hu G, Balwierz PJ, Pachkov M, Elledge SJ, van Nimwegen E, Stadler MB, Bentires-Alj M: Tyrosine phosphatase SHP2 promotes breast cancer progression and maintains tumor-initiating cells via activation of key transcription factors and a positive feedback signaling loop. Nat Med 2012, 18:529-537.

10. Yu F, Yao H, Zhu P, Zhang X, Pan Q, Gong C, Huang Y, Hu X, Su F, Lieberman J, Song E: let-7 regulates self renewal and tumorigenicity of breast cancer cells. Cell 2007, 131:1109-1123.

11. Alcalay M, Tiacci E, Bergomas R, Bigerna B, Venturini E, Minardi SP, Meani N, Diverio D, Bernard L, Tizzoni L, Volorio S, Luzi L, Colombo E, Lo Coco F, Mecucci C, Falini B, Pelicci PG: Acute myeloid leukemia bearing cytoplasmic nucleophosmin (NPMC+ AML) shows a distinct gene expression profile characterized by up-regulation of genes involved in stem-cell maintenance. Blood 2005, 106:899-902.
12. Mani SA, Guo W, Liao MJ, Eaton EN, Ayyanan A, Zhou AY, Brooks M, Reinhard F, Zhang CC, Shipitsin M, Campbell LL, Polyak K, Brisken C, Yang J, Weinberg RA: The epithelial-mesenchymal transition generates cells with properties of stem cells. Cell 2008, 133:704-715.

13. Liu S, Clouthier SG, Wicha MS: Role of microRNAs in the regulation of breast cancer stem cells. J Mammary Gland Biol Neoplasia 2012, 17:15-21.

14. Sheridan C, Kishimoto H, Fuchs RK, Mehrotra S, Bhat-Nakshatri P, Turner CH, Goulet R Jr., Badve S, Nakshatri H: CD44+/CD24- breast cancer cells exhibit enhanced invasive properties: an early step necessary for metastasis. Breast Cancer Res 2006, 8:R59.

doi:10.1186/bcr3338

Cite this article as: Wicha MS: Migratory gene expression signature predicts poor patient outcome: Are cancer stem cells to blame? Breast Cancer

Research 2012, 14:114 